\title{
Use of Anti-Mullerian Hormone (AMH) for Testing of Ovarian Reserve: A Survey of Fifteen (15) Fertility Centres in Ghana
}

\author{
Dickson Mawusi ${ }^{1,2 *}$, Michael Bright Yakass ${ }^{3,4}$, Chrissie Stancie Abaidoo5, \\ Frederick Kwaku Addai6
}

${ }^{1}$ School of Basic and Biomedical Sciences, Department of Biomedical Sciences, University of Health and Allied Sciences (UHAS), Ho, Ghana

${ }^{2}$ Airport Women's Hospital (AWH), In Vitro Fertilization (IVF) Department, Accra, Ghana

${ }^{3}$ West African Centre for Cell Biology of Infectious Pathogens (WACCBIP), Department of Biochemistry, Cell and Molecular Biology, University of Ghana, Accra, Ghana

${ }^{4}$ Assisted Conception Unit, Lister Hospital and Fertility Centre, Accra, Ghana

${ }^{5}$ School of Medicine and Dentistry, Department of Anatomy, College of Health, Kwame Nkrumah University of Science and Technology, Kumasi, Ghana

${ }^{6}$ School of Biomedical and Allied Health Sciences, Department of Anatomy, College of Health Sciences, University of Ghana Medical School, Korle Bu, Accra, Ghana

Email: *mdickson@uhas.edu.gh

How to cite this paper: Mawusi, D., Yakass, M.B., Abaidoo, C.S. and Addai, F.K. (2021) Use of Anti-Mullerian Hormone (AMH) for Testing of Ovarian Reserve: A Survey of Fifteen (15) Fertility Centres in Ghana. Advances in Reproductive Sciences, 9, 81-96.

https://doi.org/10.4236/arsci.2021.91009

Received: November 2, 2020

Accepted: January 8, 2021

Published: January 11, 2021

Copyright $\odot 2021$ by author(s) and Scientific Research Publishing Inc. This work is licensed under the Creative Commons Attribution International License (CC BY 4.0).

http://creativecommons.org/licenses/by/4.0/ (c) (i) Open Access

\begin{abstract}
Anti-Mullerian Hormone (AMH) is a dimeric glycoprotein with a molecular weight of $140 \mathrm{kD}$, encoded by a gene on the short arm of chromosome and a member of the transforming growth factor-beta (TGF- $\beta$ ) superfamily. The expression of AMH is markedly different in males and females, both in concentration and temporality. In males, Sertoli cells maintain a high concentration of AMH in utero which peaks shortly after birth and then drops precipitously at puberty. In females, granulosa cells produce very low levels of AMH in utero followed by a transient spike in the neonatal period. Concentrations of the hormone then rise steadily through adolescence to a peak in the mid-twenties and subsequently decline until becoming undetectable in menopause. The study aimed to understand how Clinicians and Clinical Embryologists used anti-mullerian hormone (AMH) test to assess ovarian reserve, direct patient selection and treatment regimens and guide in vitro fertilization (IVF) cycle management in all registered fertility hospitals in a West African country, Ghana. A web-based survey (questionnaire) using google forms was performed to solicit responses from all IVF hospitals that are registered with the Fertility Society of Ghana (FERSOG). This questionnaire consisted of fifteen (15) broader questions, ten (10) of which assessed the clinics' use of AMH. Responses were screened for quality to verify that only
\end{abstract}


one (1) survey was completed by each IVF centre. The study was conducted during May and June 2020 at the In Vitro Fertilization (IVF) Department of the Airport Women's Hospital (AWH) in Accra, Ghana. Results are reported as the proportion of IVF cycles represented by a particular answer choice. Survey responses were completed from 15 IVF centres, representing 2504 IVF cycles performed annually. A good majority (73.3\%) [1835 IVF cycles] of the respondent IVF hospitals reported to use $\mathrm{AMH}$ as a first line test and $93.3 \%$ reported it as the best test for evaluating ovarian reserve. Another $66.7 \%$ reported that $\mathrm{AMH}$ results were extremely relevant to clinical practice. However, in contrast, for predicting live birth rate, $60 \%$ reported age as the best predictor in their practice. Overall, our results indicate that AMH is considered a first line test for assessing ovarian reserve and is relevant to the clinical practice of majority of Assisted Reproductive Technologies (ART) providers in Ghana.

\section{Keywords}

Anti-Mullerian Hormone, Assisted Reproductive Technologies (ART), IVF, Ghana, Ovarian Reserve, Survey

\section{Introduction}

Most women are now postponing childbearing worldwide as a result of extensive use of contraception, desire for higher education, lack or disruption of employment, socioeconomic concerns and the growing popularity of assisted reproductive technology (ART) which has given them the impression that female fertility may be manipulated at any stage of life [1]. Human fertility on the other hand, reduces with increasing age and more so in women as with increasing age, the quality and quantity of a woman's egg pool/ovarian reserve diminish. Many women currently seeking fertility treatments are in their advance reproductive age(s). More so, the treatment seeking behaviour of sub-fertile couples in developing countries portends further delays. A recent study conducted by Hiadzi et al., 2019 [2] showed that, many sub-fertile couples start seeking fertility treatment from herbal products and associated traditional services, then through religious leaders and by the time they visit ART centres, the woman will eventually be advanced in age. Epidemiological data have consistently shown that fertility declines as early as the middle of the third decade [3] and female age remains the most important determinant of success [4] in an IVF programme. Though there is no strict definition of advanced reproductive age in women, subfertility becomes more pronounced after the age of 35 years [5] (Practice committee of ASRM, 2015). Only half of the age-related decline in fertility that occurs between 30 and 35 years and a third of that between 35 and 40 years can be overcome by IVF [3] with expected live birth rates of $25 \%-30 \%$, in women in their 20 s and 30s [3]. IVF is an invasive treatment, expensive, time consuming, stressful for patients with a relatively low to moderate success rates. Live birth rates are even 
lower (10\%) in women more than 40 years [6] and treatments with oocyte donation are likely to be effective in such cases [5].

Age of women seemed to be a better predictor of success in IVF but the emergence of the ability to measure AMH recently had added a great deal of spice into the daily practice in ART [7] and its ability to indicate ovarian reserve and predict ovarian response to stimulation has distinctly improved the planning of ovarian stimulation protocols and so increased safety and efficiency as well as aiding in the counselling of patients. AMH is a far preferred biological marker that determines the ovarian reserve in women of all ages as compared to any other basal hormonal markers [7] and for that matter IVF practitioners may prefer to have such a biological marker which is able to predict a patient's response to Controlled Ovarian hyperstimulation ( $\mathrm{COH})$.

After the birth of Louise Brown, the world's first "test-tube baby" in England in 1978 through IVF, this reproductive technology soon spread throughout Europe, North America, Australia, Asia, South America and Africa. The rapid development of IVF all over the world in the past four (4) decades has led not only to its expansion, but also to an enormous variation. Generally, sub-Saharan and Eastern Africa have fewer IVF clinics compared to all other regions in the world [8]. Within Africa, Ghana, Nigeria and South Africa, are referred to as "comparative regional success stories" [8]. In Sub-Saharan Africa (SSA), Nigeria was the first country to open an IVF centre, in 1984 [9]. The first successful IVF procedure in Ghana was conducted in 1995 at the Provita Specialist Hospital (PSH) Limited (a private hospital) in Tema, a harbour city close to the capital town Accra by Dr. Joseph Mainoo. Since then, a growing number of IVF hospitals in Ghana (more than 20), all private, have been offering IVF and other high-tech assisted reproductive technologies. To date, however, the country's public health sector is not involved in the provision of ART services. Also, the field of infertility and assisted reproductive technologies in Ghana functions without financial and insurance support from local, international health and development organizations [8] [10]. The establishment and actual functioning of IVF hospitals/clinics in Ghana is a local enterprise. On the contrary, in many ways the Ghanaian IVF industry is a highly transnational undertaking, involving an ongoing cross-border and even cross-continental flow of knowledge and ideas, skills, technologies and people [11]. While IVF has been provided in Ghana since 1995, there are no legislation or professional regulations on the use of ARTs. Thus, the hospital directors and Clinical Embryologists are those who decide on clinical and laboratory procedures and practices [12]. Furthermore, clinic success rates are neither centrally registered nor made available to the public. This may thus be described as neoliberal in Ghana's ART policy as it largely relies "on self-regulation and market forces," similar to the United States (US) which lacks a central ART policy or ART registry [13]. In 2016, the Ghana Association of Clinical Embryologists (GACE) [a professional organization for Clinical Embryologists] and thereafter, the Fertility Society of Ghana (FERSOG) [consisting of all IVF specialists and ART providers and stakeholders] have been 
established and this is leading to the creation of national regulations. Since the advent of IVF and the adjunct use of gonadotrophins, the need for tests predictive of patient response to controlled ovarian hyperstimulation $(\mathrm{COH})$ has become essential. Currently, a variety of tests (hormonal profiling) can be used to estimate a woman's ovarian reserve, or the reproductive potential of the oocytes remaining within the ovary. Ovarian reserve testing modalities include antral follicle count (AFC), ovarian volume, early follicular phase follicle stimulation hormone $(\mathrm{FSH})$ and oestradiol $\left(\mathrm{E}_{2}\right)$ levels, inhibin $\mathrm{B}$, the clomiphene citrate (CC) challenge test, and anti-mullerian hormone [14]. However, identifying which of these tests are most accurate in predicting the ovarian response to $\mathrm{COH}$ and the potential for predicting pregnancy is yet to be definitively established. As a result, practitioners currently rely on a variety of different tests as part of their infertility evaluation and to predict ovarian response to $\mathrm{COH}$ [15] [16]. AMH is one of the most recent tests developed to measure ovarian reserve. AMH is a glycoprotein belonging to the beta-transforming growth factor (B-TGF) super family secreted from the granulosa cells of small ovarian follicles [17]. In the developing female human embryo, the absence of AMH allows the mullerian ducts to differentiate into the upper portion of the vagina, cervix, uterus, and fallopian tubes [18]. However, as early as 36 weeks gestation, female foetuses begin producing AMH, which steadily increases in production until follicles reach the antral phase [19]. With increasing age, there is a steady decline in levels of AMH until it becomes undetectable, which correlates with the onset of menopause [20]. This rise and fall of the AMH level correspond with the number of oocytes remaining in the ovary [21]. Given that AMH represents the pool of oocytes remaining in the ovary, as a metric, it results in both consistent inter- and intra-menstrual cycle measurements [22]. AMH testing, as a measure of ovarian reserve is recommended (ASRM and ESHRE) and routinely used in ART practice in Europe and North America. Although committee opinions from major organizations support the routine use of $\mathrm{AMH}$, it is unclear what proportion of IVF centres in Ghana are currently using AMH to guide IVF cycle management in relation to other metrics of ovarian reserve (basal hormone tests). There are lots of researched and published works about the use of AMH in developed countries, however, no prior study has attempted to assess ART practitioners' perceptions and practice patterns with regard to the use of AMH in Ghana. Currently, there is no data regarding how AMH is been used by IVF practitioners in the country. The aim of this study is to use a survey (designed questionnaire using google forms) to obtain a first-hand information from all registered IVF clinics with FERSOG and provide insight on how AMH is currently applied to IVF cycle management and the measure of ovarian reserve in Ghana.

\section{Materials and Methods}

\section{Ethical Consideration}

The aim of the study and the procedure for answering the questionnaire was explained to all participants. 
Ethical Clearance was obtained from the Committee on Human Research, Publication and Ethics (CHRPE) of the School of Medicine and Dentistry, Department of Anatomy, College of Health, Kwame Nkrumah University of Science and Technology (KNUST). Written consent was obtained from all participants.

\section{Survey Content}

A 5-item questionnaire (5 questions) collected the demographics of the responding IVF centres and 10 other questions assessed the clinical applications of $\mathrm{AMH}$ to IVF cycle management and the evaluation of ovarian reserve. For each question, multiple choice answers were provided from which only a single answer could be selected. This survey questionnaire was made available via contact email addresses of the hospitals from May 01 to June 01, 2020. All registered IVF centres with FERSOG were invited by an e-mail message to participate in completing the survey. The survey contained a demographic section and a medical section that evaluated the practice patterns and opinions of respondents with a series of multiple-choice questions.

\subsection{Calculation of the Minimum Sample Size}

We used the rate of using AMH test to assess ovarian reserve in fertility hospitals of $50 \%$ in order to determine the minimum sample size.

The sample size was calculated using the formula:

$$
n=\frac{Z^{2} P(1-P)}{m^{2}}
$$

where $Z=$ The physical value, $P=50 \%$ is the rate of using AMH to assess ovarian reserve to direct patient selection and treatment regimens, $m=$ margin of error (0.001). This gave the minimum, $n$, of 2504 IVF cycles performed annually from the survey responses completed from 15 IVF centres in Ghana.

\subsection{Quality Control of Collected Data}

In order to minimize duplicate reports from a clinic and possible data errors, five (5) demographic parameters were used to crosscheck the demographic information submitted by the survey respondent with the existing registration information for that clinic with FERSOG secretariat. These parameters included the name of the clinic, the name of the hospital director and e-mail address. If at least three of these parameters matched the information previously registered, then the results from the reporting centre are then included in the statistical analyses.

\subsection{Statistical Analyses}

The analysis was based on the number of IVF cycles reported by the unit and not on the number of clinics in the study. For each question, the survey provided multiple choices from which only a single answer could be chosen. The results of 
the multiple-choice questions are reported as both the proportion of the total number of IVF cycles represented by that particular answer choice (respondent-IVF cycles) and as the proportion of clinics which selected that answer choice.

\section{Results}

Completed electronic survey forms were received from 15 IVF hospitals which performed a total of 2504 IVF cycles annually, from 3 regions in Ghana (Greater Accra, Ashanti and Western). The response rate for survey completion was $100 \%$ of the 15 clinics registered with FERSOG. The geographic location and the percent contribution to the total survey response was divided into 3 regions: Greater Accra (1366 cycles [54.55\%], 11 IVF centres [73.33\%]), Ashanti (1105 cycles [44.13\%], 3 IVF centres [20\%]) and Western (33 cycles [1.32\%], 1 IVF centre [6.67\%]). Overall, 66.7\% (1670 cycles) of respondent-IVF centres reported that the majority of patients visiting their IVF hospitals underwent ovarian reserve testing. Ninety-three-point three percent [93.3\%] (2336 cycles) reported that $\mathrm{AMH}$ is the best test for evaluating ovarian reserve, while 6.7\% (168 cycles) reported that AFC is the best test (Table 1). Another 6.7\% (168 cycles) reported that basal hormone testing is the best test for evaluating ovarian reserve (Table 2). When asked which factor or test best predicts ongoing pregnancy rate, $60.0 \%$ (1502 cycles) reported age, 26.6\% (669 cycles) reported AMH, and $13.3 \%$ (333) reported other (Table 1). When asked which test is best for evaluating ovarian reserve, $88.9 \%$ (2226 cycles) of respondent-IVF centres represented by selecting a specific test to evaluate ovarian reserve responded yes (Figure 1). When asked if respondents consider AMH as a first- or second-line test of ovarian reserve, $73.3 \%$ (1835 cycles) of respondent-IVF cycles reported it as a first line test (Table 2). When respondents were asked in their opinion, what is the best use of AMH, 94.4\% (2364 cycles) of respondent, IVF cycles reported it to predict both low and high ovarian reserve and response to stimulation. The same results where noted when the influence of the cost to perform an AMH test was removed as a consideration for performing the test. Regarding the clinical application of AMH, 66.7\% (1670 cycles) of respondent-IVF cycles reported it as either extremely relevant while $33.3 \%$ (834) considered it as not relevant. When respondents were asked what single test is best for choosing a gonadotropin dose, AMH [60\% (1502 cycles)] was selected over AFC, 13.3\% (333 cycles), FSH, 6.7\% (168 cycles and age, 20.0\% (501 cycles) respectively. Also, respondents were asked how best to use AMH, majority (93.3\%; $2336 \mathrm{cy}-$ cles) reported to use it to predict both low and high ovarian reserve. Finally, majority of respondent-IVF centres, 93.3.\% (2336 cycles) reported that they would stimulate patients based on the AMH levels. As resource availability and practice patterns may vary between socio-geographic areas in the regions in Ghana, the above listed survey responses were again separated into the three different geographic areas: Greater Accra, Ashanti and Western (Table 3). 
When asked if AMH is considered a first line test, $53.33 \%(8 / 15)$ of respondent-IVF centres from Greater Accra region reported yes, $13.33 \%(2 / 15)$ reported yes from Ashanti and 1.67\% (1/15) reported yes from Western (Table 3). Similarly, 66.67\% (10/15) of respondent-IVF centres from Greater Accra, 6.67\% (1/15) from Ashanti and 6.67\% (1/15) from Western region would choose AMH for assigning gonadotropin dosages above other ovarian reserve test choices (age, FSH, AFC). Finally, the question with the highest similarity between geographic regions was the low selection of AMH $(26.7 \%$ [4/15]) as the best predictor of ongoing pregnancy rates. Majority of IVF centres in the 3 regions essentially agreed that $\mathrm{AMH}$ was not the best predictor of ongoing pregnancy rates but rather the age $(60 \%[9 / 15])$ of the patient (Table 1$)$.

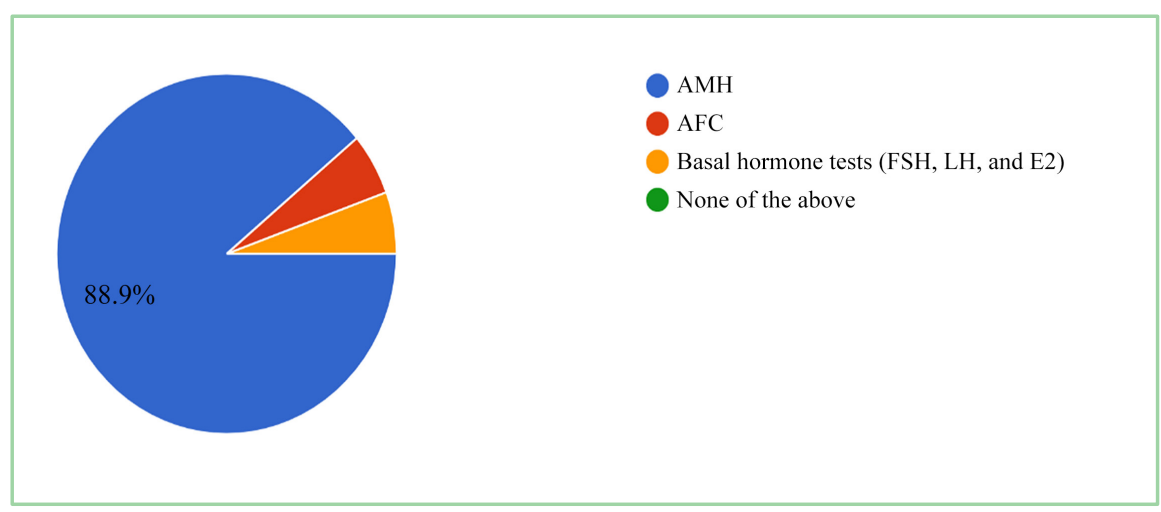

Figure 1. Demonstrates the proportion of respondent-IVF centres represented by selecting a specific test to evaluate ovarian reserve in response to the question "Which test do you think is best for evaluating ovarian reserve?"

Table 1. AMH as a favoured biological marker for predicting of ovarian reserve as compared to other parameters.

\begin{tabular}{|c|c|c|c|c|c|c|c|c|c|c|}
\hline Parameter & Age & & FSH & & AFC & & $\mathrm{AMH}$ & & Other & \\
\hline & $\begin{array}{c}\text { Centres \% } \\
(n)\end{array}$ & $\begin{array}{c}\text { No. } \\
\text { Cycles \% } \\
(n)\end{array}$ & $\begin{array}{c}\text { Centres } \% \\
(n)\end{array}$ & $\begin{array}{c}\text { No. } \\
\text { Cycles \% } \\
(n)\end{array}$ & $\begin{array}{c}\text { Centres \% } \\
(n)\end{array}$ & $\begin{array}{c}\text { No. } \\
\text { Cycles \% } \\
(n)\end{array}$ & $\begin{array}{c}\text { Centres \% } \\
(n)\end{array}$ & $\begin{array}{c}\text { No. } \\
\text { Cycles \% } \\
(n)\end{array}$ & $\begin{array}{c}\text { Centres \% } \\
(n)\end{array}$ & $\begin{array}{c}\text { No. } \\
\text { Cycles \% } \\
(n)\end{array}$ \\
\hline Evaluating ovarian reserve & - & - & $0(0)$ & $0(0)$ & $6.7(1)$ & $6.7(168)$ & $93.3(14$ & $93.3(2336)$ & - & - \\
\hline Guide as $\mathrm{COH}$ starting dose & $20.0(3)$ & $20.0(501)$ & $6.6(1)$ & $6.7(168)$ & $13.3(2)$ & $13.3(333)$ & $60.0(9)$ & $60.0(1502)$ & - & - \\
\hline Estimation of ovarian failure & - & - & - & - & $13.3(2)$ & $13.3(333)$ & $86.7(13)$ & $86.7(2171)$ & - & - \\
\hline $\begin{array}{l}\text { Best predictor of ongoing } \\
\text { pregnancy in clinic }\end{array}$ & $60.0(9)$ & $60.0(1502)$ & - & - & - & - & $26.7(4)$ & $26.7(669)$ & $13.3(2)$ & $13.3(333)$ \\
\hline
\end{tabular}

Table 2. Use of $\mathrm{AMH}$ as a first line ovarian function test.

\begin{tabular}{ccccc}
\hline & \multicolumn{2}{c}{ YES } & NO \\
\hline Parameter & IVF centres $\%(n)$ & IVF Cycles $\%(n)$ & IVF centres $\%(n)$ & IVFCycles $\%(n)$ \\
\hline Perform ovarian function testing & $66.7(10)$ & $66.7(1670)$ & $33.3(5)$ & $33.3(834)$ \\
AMH as first-line testing & $73.3(11)$ & $73.3(1835)$ & $26.7(4)$ & $26.7(669)$ \\
Base COH protocol on AMH & $93.3(14)$ & $93.3(2336)$ & $6.7(1)$ & $6.7(168)$ \\
\hline
\end{tabular}


Table 3. Regional/geographical distribution of fertility centres that used AMH and are registered with FERSOG in Ghana.

\begin{tabular}{ccccc}
\hline Name of region & IVF centres $\%(n)$ & No. Cycles $\%(n)$ & $\begin{array}{c}\text { AMH as a first line } \\
\text { test } \%(n)\end{array}$ & $\begin{array}{c}\text { AMH as the best marker for assigning } \\
\text { the starting gonadotropin dose \% (n) }\end{array}$ \\
\hline Greater Accra & $54.55(11)$ & $54.55(1366)$ & $53.33(8)$ & $66.67(10)$ \\
Ashanti & $44.13(3)$ & $44.13(1105)$ & $13.33(2)$ & $6.67(1)$ \\
Western & $1.32(1)$ & $1.32(33)$ & $6.67(1)$ & $6.67(1)$ \\
Total & 15 & $100(2504)$ & $73.33(11)$ & $80.0(12)$ \\
\hline
\end{tabular}

\section{Discussion}

This study surveyed the practice of embryologists and clinicians in fifteen (15) fertility centres evaluating the use of AMH and its application to IVF in Ghana. Currently, IVF hospitals or clinics have multiple tests for evaluating ovarian reserve and estimating the potential response to $\mathrm{COH}$. Based on our survey results, AMH appears to be a highly valued test with an important role in both evaluating ovarian reserve and attempting to predict ovarian response to stimulation with $73.3 \%$ (11/15) of responding practitioners reporting it as a first line test and 93.3\% (14/15) reporting that it is the best test for evaluating ovarian reserve in their practice. In 2008, Maheshwari et al. [23] reported on a survey results from 72 IVF providers within the UK's National Health Service with the goal of ascertaining how IVF was offered to women of advanced maternal age and how ovarian reserve was assessed. They identified that $95 \%$ of IVF centres used baseline FSH to evaluate ovarian reserve and only $4.5 \%$ used AMH. Of note: this study's data was collected from 2006 to 2007, and clinical practice patterns within the IVF community continually changed. Given the time lapse from 2006-2007 until May to June 2020 (the year of our study), this study likely does not accurately reflect the current use of AMH within the UK. Thus, as illustrated by this contrast, AMH use has dramatically increased from 2006 to present (2020). A previous study by Van Voorhis and colleagues (Van Voorhis, 2010), surveyed clinics within the USA with the highest reported IVF live birth rates to assess for specific practice patterns that contributed to the IVF success. They identified specific practices common to high-performing clinics which included ovarian reserve screening for all patients and the use of cycle day $3 \mathrm{FSH}$ and AFC. With specific regard to AMH, they reported that only $30 \%$ of high performing clinics used $\mathrm{AMH}$ to measure ovarian reserve [24]. In this study, we report that, $66.7 \%$ (1670 cycles (10/15) of respondent-IVF cycles] of reporting clinics test all IVF patients for ovarian reserve.

Whether all patients require ovarian reserve testing prior to IVF however remains unanswered. Although fertility predictably declines with age, the outcomes of infertility treatment vary greatly among patients within the same age cohorts. Ovarian reserve testing may assist IVF providers in identifying those patients at risk for suboptimal outcomes and provide information to more effectively counsel and select a gonadotropin dose for the patient undergoing $\mathrm{COH}$. However, when ovarian reserve testing is applied broadly, especially to young 
women, the potential for a false positive result identifying low ovarian reserve increases and lowers the predictive power of the test, and may be misleading [25]. In a recent ASRM committee opinion, the authors concluded that evidence of decreased ovarian reserve does not necessarily equate inability to conceive [14] [25] [26] [27]. However, the ASRM committee opinion does not discourage the broad use of AMH as there is likely benefit and conclude by stating that "there is insufficient evidence to recommend that any ovarian reserve test now available should be used as a sole criterion for the use of assisted reproductive technologies" [14]. Additionally, a consensus opinion from ASRM and ESHRE stated that "ovarian reserve testing has moderate accuracy in predicting quantitative responses but low accuracy for qualitative predictions, unless very high thresholds are used" [16]. At this juncture, no definitive statement that all IVF patients should undergo ovarian reserve testing has been made from the two largest reproductive societies in the world [thus; ESHRE and ASRM].

When the respondents in our research were asked if AMH is considered a first line test and if they believed AMH was the best test for evaluating ovarian reserve, the response was $73.3 \%$ and $93.3 \%$ respectively. Multiple analyses comparing the various ovarian reserve tests have been performed with no clear consensus on selecting a superior test [26] [28]; however, AMH and AFC appear to be favoured by IVF practitioners [28] [29] [30]. Prior researchers have attempted to address the precision, accuracy, and predictive value of AMH in addressing those outcomes, comparing AMH both individually and in combination with other tests. In the past, the uses of early (Day 2/3) follicular FSH serum levels were considered the gold standard for assessing ovarian reserve; however, multiple other metrics have since emerged with robust studies supporting their use. Using baseline FSH levels, linear correlations demonstrate that rising FSH values correspond with decreasing AMH values; however, predictive superiority for actual IVF outcomes has yet to be coordinated [31]. When a single marker is selected, AMH appears to outperform other metrics of ovarian reserve in predicting oocytes retrieved and the number of cancelled cycles [30] although there are contrasting reports [30]. This is especially seen with analysing pregnancy rates and live births. In several series, AMH levels are associated with live births [28] [32]; but in others, no association was identified [33]. In a recent 2015 meta-analysis conducted by Tal et al., [34] 19 studies were analysed by specific subpopulations comparing the predictive value of AMH with implantation and pregnancy rates, and they concluded that $\mathrm{AMH}$ has some association, but with weak predictive value [34]. In another recent meta-analysis, which included 13 studies, they also conclude that AMH does have some association with predicting live birth; however, its predictive accuracy is poor with low calculated diagnostic odds ratios [35]. Despite the confounding results identified within the literature regarding the predictive value for pregnancy outcomes, $\mathrm{AMH}$ as a measure of ovarian reserve is generally reported as uniformly accurate. However, the hesitance to fully adopt AMH into clinical practice demonstrated by several researchers (including our study) may have a historical basis on the development of the AMH assays 
integrated into clinical use [36]. Confounding the clinical use of AMH from 2002 till now [July, 2020] is the availability of different commercial assays, which resulted in substantially different quantitative values [37] [38]. Since 2011, studies reporting on $\mathrm{AMH}$ values have been measured by the AMH Gen II assay and thus lead to an improvement in the homogeneity of the AMH literature.

The predictive value of AMH on IVF outcomes is still yet to be definitively established and multiple reviews addressing the use of AMH have outlined both actual and theoretical shortcomings of its application to fertility treatments. With newer, more reliable AMH assays available, the need for relevant nomograms and cut-off values related to treatment outcomes should be forthcoming but currently are still lacking. Given that the exact role of AMH is yet to be definitively established, our data provide an interesting perspective that despite the potential shortcomings of AMH and the current evolution of the assays used, it is still considered a first line test of ovarian reserve and is reported by the majority of our respondents to have significant direct value in the management of IVF cycles. Despite the encouraging recent reports of AMH use and the widespread use demonstrated by other scientists and this study, providers must be cognoscente of the limitations; especially if it is used as the primary test to evaluate ovarian reserve. First, the AMH values produced by the specific assay should be considered unique to the laboratory completing the assay. As discussed above, Gen II AMH assays should be used making comparing values obtained with current literature more relevant. However, even with the same Gen II assay, a wide range of average values were still reported by Zuvela et al. [39] [40] [41]. Additionally, caution must be used if results from a single ovarian reserve marker will lead to specific, definitive IVF treatment alteration. This is especially important that IVF centres use caution not to deny patients autologous oocyte(s) use based on a single AMH value. AMH has demonstrated benefits with assigning gonadotropin dosing, avoiding cycle cancellation and OHSS.

\section{Limitation of the Study}

Although this study represents the first of its kind in Ghana, a potential weakness of respondent bias or incorrectly classifying their clinical IVF cycles may exist. Additionally, our findings represent the opinions of IVF centres, and do not necessarily represent best clinical practices or evidence-based medicine.

\section{Conclusions}

This study provides insight into the use of $\mathrm{AMH}$ and its value relative to other metrics of ovarian reserve from a small population of IVF centres in Ghana. At this time, it appears that the vast majority of ART practitioners are using AMH regularly in clinical practice and place a high value on its ability to evaluate ovarian reserve and its predictive abilities for $\mathrm{COH}$.

Dickson Mawusi (DM) designed the questionnaire and study and it was reviewed by Professor Chrissie Stancie Abaidoo (CSA) and Professor Frederick 
Kwaku Addai. DM and Michael Bright Yakass (MBY) analysed the data. DM wrote the manuscript. All authors have critically reviewed and proofread the manuscript and every mistake or error has been corrected.

\section{Acknowledgements}

The authors would like to thank all registered members of FERSOG for their responses and participation

\section{Conflicts of Interest}

The authors declare no conflicts of interest regarding the publication of this paper.

\section{References}

[1] Bradley, S.E.K., Chelsea, B.P., Akinrinola, B. and Trevor, C. (2019) Global Contraceptive Failure Rates: Who Is Most at Risk? Studies in Family Planning, 50, 3-24. https://doi.org/10.1111/sifp.12085

[2] Hiadzi, R.A. and Bryan, J.W. (2019) Infertility Treatment Decision-Making in Ghana and Contestations That May Arise: A Prospective Sociological Study. Global Reproductive Health, 4, e32. https://doi.org/10.1097/GRH.0000000000000032

[3] Leridon, H. (2004) Can Assisted Reproduction Technology Compensate for the Natural Decline in Fertility with Age? A Model Assessment. Human Reproduction, 19, 1548-1553. https://doi.org/10.1093/humrep/deh304

[4] Moolhuijsen, L.M.E. and Visser, J.A. (2020) Anti-Müllerian Hormone and Ovarian Reserve: Update on Assessing Ovarian Function. The Journal of Clinical Endocrinology \& Metabolism, 105, 3361-3373.

[5] Practice Committee of the American Society for Reproductive Medicine (ASRM) (2015) Diagnostic Evaluation of the Infertile Female: A Committee Opinion. Fertility and Sterility, 103, e44-e50. https://doi.org/10.1016/j.fertnstert.2015.03.019

[6] Tomlinson, M.J., Harbottle, J.S., Woodward, B.J. and Lindsay, K.S. (2012) Association of Biomedical Andrologists-Laboratory Andrology Guidelines for Good Practice. Human Fertility, 3, 156-173. https://doi.org/10.3109/14647273.2012.747888

[7] Lehmann, P., Maria P.V., Julio, S., Louise, P., Wael, J., François, B., Simo, P. and Isaac-Jacques, K. (2014) Anti-Müllerian Hormone (AMH): A Reliable Biomarker of Oocyte Quality in IVF. Journal of Assisted Reproduction and Genetic, 31, 493-498. https://doi.org/10.1007/s10815-014-0193-4

[8] Inhorn, M.C. and Patrizio, P. (2015) Infertility around the Globe: New Thinking on Gender, Reproductive Technologies and Global Movements in the 21st Century. Human Reproduction, 21, 411-426. https://doi.org/10.1093/humupd/dmv016

[9] Ombelet, W. (2007) Affordable IVF for Developing Countries. Reproductive BioMedicine Online, 3, 257-265. https://doi.org/10.1016/S1472-6483(10)60337-9

[10] Hörbst, V. and Wolf, A. (2014) ARV and ART: Medicoscapes and the Unequal Place-Making for Biomedical Treatments in Sub-Saharan Africa. Medical Anthropology Quarterly, 28, 182-202. https://doi.org/10.1111/maq.12091

[11] Dilger, H., Kane, A. and Langwick, S.A. (2012) Medicine, Mobility, and Power in Global Africa: Transnational Health and Healing. Indiana University Press, Bloomington, 2, 30-32.

[12] Hörbst, V. and Gerrits, T. (2015) Transnational Connections of Health Profession- 
als: Medicoscapes and Assisted Reproduction in Ghana and Uganda. Ethnicity and Health, 21, 357-374. https://doi.org/10.1080/13557858.2015.1105184

[13] Martin, L.J. (2009) Reproductive Tourism in the Age of Globalization. Globalizations, 6, 249-263. https://doi.org/10.1080/14747730802500398

[14] Practice Committee of the American Society for Reproductive Medicine (ASRM) (2015) Testing and Interpreting Measures of Ovarian Reserve: A Committee Opinion. Fertility and Sterility, 103, e9-e17. https://doi.org/10.1016/j.fertnstert.2014.12.093

[15] Ferraretti, A.P. and Gianaroli, L. (2014) The Bologna Criteria for the Definition of Poor Ovarian Responders: Is There a Need for Revision? Human Reproduction, 29, 1842-1845. https://doi.org/10.1093/humrep/deu139

[16] Gianaroli, L. (2012) Best Practices of ASRM and ESHRE: A Journey through Reproductive Medicine. Fertility and Sterility, 98, 1380-1394. https://doi.org/10.1016/j.fertnstert.2012.07.1164

[17] Seifer, D.B. and Maclaughlin, D.T. (2007) Mullerian Inhibiting Substance Is an Ovarian Growth Factor of Emerging Clinical Significance. Fertility and Sterility, 88, 539-546. https://doi.org/10.1016/j.fertnstert.2007.02.014

[18] Silva, M.S.B. and Giacobini, P. (2020) New Insights into Anti-Müllerian Hormone Role in the Hypothalamic-Pituitary-Gonadal Axis and Neuroendocrine Development. Cellular and Molecular Life Sciences. https://doi.org/10.1007/s00018-020-03576-x

[19] Catherine, D., McCormack, Shalem, Y.L., Denise, L.F., Gustaaf, A.D. and Claire, T.R. (2019) Anti-Müllerian Hormone Levels in Recurrent Embryonic Miscarriage Patients Are Frequently Abnormal, and May Affect Pregnancy Outcomes. Journal of Obstetrics and Gynaecology, 39, 623-627. https://doi.org/10.1080/01443615.2018.1552669

[20] Broer, S.L. (2011) Anti-Mullerian Hormone Predicts Menopause: A Long-Term Follow-Up Study in Normoovulatory Women. Journal of Clinical Endocrinology and Metabolism, 96, 2532-2539. https://doi.org/10.1210/jc.2010-2776

[21] Hansen, K.R. (2011) Correlation of Ovarian Reserve Tests with Histologically Determined Primordial Follicle Number. Fertility and Sterility, 95, 170-175.

https://doi.org/10.1016/j.fertnstert.2010.04.006

[22] van Disseldorp (2010) Comparison of Inter- and Intra-Cycle Variability of Anti Mullerian Hormone and Antral Follicle Counts. Human Reproduction, 25, 221-227. https://doi.org/10.1093/humrep/dep366

[23] Maheshwari, A., Hamilton, M. and Bhattacharya, S. (2008) A Survey of Clinicians' Views on Age and Access to IVF and the Use of Tests of Ovarian Reserve Prior to IVF in the United Kingdom. Human Fertility, 11, 23-27. https://doi.org/10.1080/14647270701541095

[24] Van Voorhis, B.J. (2010) What Do Consistently High-Performing in Vitro Fertilization Programs in the U.S. Do? Fertility and Sterility, 94, 1346-1349. https://doi.org/10.1016/j.fertnstert.2010.06.048

[25] Barnhart, K. and Osheroff, J. (1998) Follicle Stimulating Hormone as a Predictor of Fertility. Current Opinion in Obstetrics and Gynaecology, 10, 227-232. https://doi.org/10.1097/00001703-199806000-00009

[26] Broekmans, F.J. (2006) A Systematic Review of Tests Predicting Ovarian Reserve and IVF Outcome. Human Reproduction Update, 12, 685-718.

https://doi.org/10.1093/humupd/dml034 
[27] Esposito, M.A., Coutifaris, C. and Barnhart, K.T.A. (2002) Moderately Elevated Day 3 FSH Concentration Has Limited Predictive Value, Especially in Younger Women. Human Reproduction, 17, 118-223. https://doi.org/10.1093/humrep/17.1.118

[28] Eldar-Geva, T. (2005) Dynamic Assays of Inhibin B, Anti-Mullerian Hormone and Estradiol Following FSH Stimulation and Ovarian Ultrasonography as Predictors of IVF Outcome. Human Reproduction, 20, 3178-3183. https://doi.org/10.1093/humrep/dei203

[29] Nardo, L.G. (2009) Circulating Basal Anti-Mullerian Hormone Levels as Predictor of Ovarian Response in Women Undergoing Ovarian Stimulation for in Vitro Fertilization. Fertility and Sterility, 92, 1586-1593. https://doi.org/10.1016/j.fertnstert.2008.08.127

[30] La Marca, A. (2010) Anti-Mullerian Hormone (AMH) as a Predictive Marker in Assisted Reproductive Technology (ART). Human Reproduction Update, 16, 113-130. https://doi.org/10.1093/humupd/dmp036

[31] Singer, T. (2009) Correlation of Antimullerian Hormone and Baseline Follicle-Stimulating Hormone Levels. Fertility and Sterility, 91, 2616-2619.

https://doi.org/10.1016/j.fertnstert.2008.03.034

[32] Nelson, S.M., Yates, R.W. and Fleming, R. (2007) Serum Anti-Mullerian Hormone and FSH: Prediction of Live Birth and Extremes of Response in Stimulated Cycles-Implications for Individualization of Therapy. Human Reproduction, 22, 2414-2421. https://doi.org/10.1093/humrep/dem204

[33] Smeenk, J.M. (2007) Antimullerian Hormone Predicts Ovarian Responsiveness, But Not Embryo Quality or Pregnancy, after in Vitro Fertilization or Intracytoplasmic Sperm Injection. Fertility and Sterility, 87, 223-226. https://doi.org/10.1016/j.fertnstert.2006.06.019

[34] Tal, R. (2015) Antimullerian Hormone as Predictor of Implantation and Clinical Pregnancy after Assisted Conception: A Systematic Review and Meta-Analysis. Fertility and Sterility, 103, 119-130. https://doi.org/10.1016/j.fertnstert.2014.09.041

[35] Iliodromiti, S. (2014) The Predictive Accuracy of Anti-Mullerian Hormone for Live Birth after Assisted Conception: A Systematic Review and Meta-Analysis of the Literature. Human Reproduction Update, 20, 560-570.

https://doi.org/10.1093/humupd/dmu003

[36] Schipper, I. (2012) Limitations and Pitfalls of Antimullerian Hormone Measurements. Fertility and Sterility, 98, 823-824. https://doi.org/10.1016/j.fertnstert.2012.07.1105

[37] Broer, S.L. (2014) Anti-Mullerian Hormone: Ovarian Reserve Testing and Its Potential Clinical Implications. Human Reproduction Update, 20, 688-701.

https://doi.org/10.1093/humupd/dmu020

[38] Nelson, S.M. and La Marca, A. (2011) The Journey from the Old to the New AMH Assay: How to Avoid Getting Lost in the Values. Reproductive Biomedicine Online, 23, 411-420. https://doi.org/10.1016/j.rbmo.2011.06.011

[39] Freour, T. (2007) Measurement of Serum Anti-Mullerian Hormone by Beckman Coulter ELISA and DSL ELISA: Comparison and Relevance in Assisted Reproduction Technology (ART). Clinica Chimica Acta, 375, 162-164. https://doi.org/10.1016/j.cca.2006.06.013

[40] Rustamov, O. (2012) Anti-Mullerian Hormone: Poor Assay Reproducibility in a Large Cohort of Subjects Suggests Sample Instability. Human Reproduction, 27, 3085-3091. https://doi.org/10.1093/humrep/des260 
[41] Zuvela, E., Walls, M. and Matson, P. (2013) Within-Laboratory and Between-Laboratory Variability in the Measurement of Antimullerian Hormone Determined within an External Quality Assurance Scheme. Reproductive Biology, 13, 255-257. https://doi.org/10.1016/j.repbio.2013.04.005 


\section{Appendix I. Anti-Mullerian Hormone (AMH) Research Questionnaire in Ghana}

\section{Contact Information:}

Dickson Mawusi

University of Health and Allied Sciences (UHAS)

Department of Biomedical Sciences

Phone: 0244297814

Email: mdickson@uhas.edu.gh

The aim of this study is to assess how Anti-Mullerian hormone (AMH) is used across fertility centres in Ghana to test ovarian reserve and guide in in vitro fertilization (IVF) cycle management.

Part I - Demographic section

$>$ Name of Hospital/Clinic:

Name of Medical Director:

Name of Clinical Embryologist(s):

$>$ Email address:

Is your facility registered with the Fertility Society of Ghana (FERSOG)? Yes/No

$>$ Are you a registered member of the fertility Society of Ghana (FERSOG)? Yes/No

- How many IVF cycles has your hospital/Clinic performed annually during the past 3 years?

\section{Part II}

For each question, multiple choice answers are provided. Please select by circling ONLY one answer.

1) Have majority of patients visiting your IVF clinic already undergone ovarian function testing?

- Yes

- No

- I do not know

2) Would you consider AMH testing a first- or second-line test?

- First

- Second

- I do not know

3) Would you change your stimulation protocol based on AMH results?

- Yes

- No

- I do not know

4) How relevant do you consider the use of $\mathrm{AMH}$ testing in your routine/practice?

- Extremely relevant

- Relevant

- Not so relevant

- Uninteresting 
5) If the AMH test is available in your hospital/clinic at no cost, would you use it routinely?

- Yes

- No

- I have not thought about this

6) If you have to choose one of the factors listed below, which would serve you best in assigning the starting gonadotropin dose?

- Age

- $\mathrm{FSH}$

- $\mathrm{AFC}$

- $\mathrm{AMH}$

7) Which test do you think is best for evaluating ovarian reserve?

- $\mathrm{AMH}$

- $\mathrm{AFC}$

- Basal hormone tests (FSH, LH, and E2)

- None of the above

8) In your opinion, what is the best use of AMH?

- To predict low ovarian reserve and response to stimulation

- To predict high ovarian reserve and response to stimulation

- To predict both low and high ovarian reserve and response to stimulation

- To predict pregnancy and live birth rates

- None of the above

9) Which test do you think is best predictor for premature ovarian failure or menopause?

- $\mathrm{AMH}$

- AFC

- Basal hormone tests (FSH, LH, and E2)

- None of the above

10) What do you think is the best predictor of ongoing pregnancy rate in your Clinic/Hospital?

- Age

- $\mathrm{FSH}$

- $\mathrm{AFC}$

- $\mathrm{AMH}$

- Other 